\title{
Advanced Monitoring of Cold Chain using Wireless Sensor Network and Sensor Cloud Infrastructure
}

\author{
Abel Avitesh Chandra ${ }^{1, *}$, Seong-Ro Lee ${ }^{2}$ \\ Department of Electronics Engineering, Mokpo National University, South Korea; E-Mails: \\ abelavit@yahoo.com; srlee@mokpo.ac.kr \\ * Abel Avitesh Chandra; E-Mail: abelavit@yahoo.com;
}

Published: 1 June 2014

\begin{abstract}
The Internet of Things paradigm is a new research field which connects the physical world objects to the Internet and allowing easy access to these objects in order to monitor and manage them. The objects are associated with unique identifiers and capability to transfer data over network without the intervention of humans and traditional computers. Wireless sensor networks play a major role in this paradigm in relating the physical object data to the Internet. The wireless sensor networks are put together using low cost computing devices and embedded systems such as Arduino, Raspberry Pi and other RF systems. The Sensor cloud is a secondary form of cloud computing which enables the management of the physical world objects on cloud with features to store, process, visualize and share data from these objects. The sensor cloud is therefore becoming popular in providing an open, flexible and reconfigurable platform for many monitoring and controlling applications. This paper explores the idea of IoT to enable the monitoring of cold supply chain through the deployment of wireless sensor network in logistics and cold storage facilities and integrating them to the Xively sensor cloud for a complete monitoring and end-to-end visibility.
\end{abstract}

Keywords: Internet of Things; Wireless Sensor Network; Sensor Cloud; Cold Chain

\section{Introduction}

Cold chain is the process of maintaining optimal conditions during transport, storage and handling of temperature sensitive products, starting at the manufacturer and ending with the administration of product to the client. The focus of cold chain management is on pharmaceutical products, chemicals 
and groceries. The primary goal of cold chain management is to optimize the product quality and product safety and minimization of wastage [1].

Vaccines are sensitive biological products which can become less effective or even destroyed when exposed to temperatures outside the recommended range [2]. The recommended temperature range is between $+2^{\circ} \mathrm{C}$ and $+8^{\circ} \mathrm{C}$ for refrigerated vaccines and optimum temperature of $-15^{\circ} \mathrm{C}$ for frozen vaccines. The exposure leads in some loss of potency with each episode of exposure and repetitive exposure results in a cumulative loss of potency that is not reversible.

There are several reasons for maintaining the potency of vaccines. The product being used has to be effective otherwise it may result in re-emergence or occurrence of vaccine preventable diseases. The management of resources is important as vaccines are expensive and can be short in supply. Moreover, revaccination of patients who receive ineffective vaccine is professionally uncomfortable and may cause a loss of public confidence in health care systems.

Three main elements combine to ensure proper product transport, storage and handling. These are: trained personnel, transport and storage equipment, efficient management procedures. This paper in the following sections looks at how transport and storage equipments as well as the management procedures can be optimized in order to deliver a safer cold chain.

\section{Architecture Design for Cold Chain Monitoring}

The project's operation is governed by the functionality of the Internet of Things [3]. For this idea to be realized, two architectures are required: the sensing entity in the environment which collects data and the cloud service where those data are stored. Our cold chain monitoring system is based on a network of connected sensors which collect data and upload it to the cloud from which the data is accessible from anywhere.

The monitoring system is further divided into two subsystems. One for monitoring cold storage environment at facilities whiles the other for monitoring in logistics. The overall system is described in Figure 1 which shows the conceptualization of the two subsystems.

Figure 1. Monitoring setup at (a) Storage facility. (b) Transport facility.

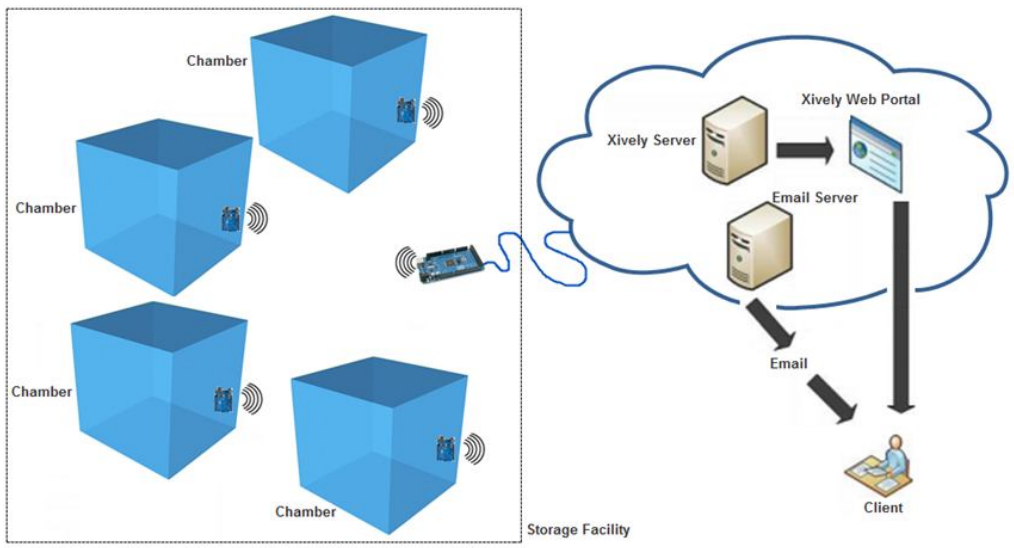

(a)

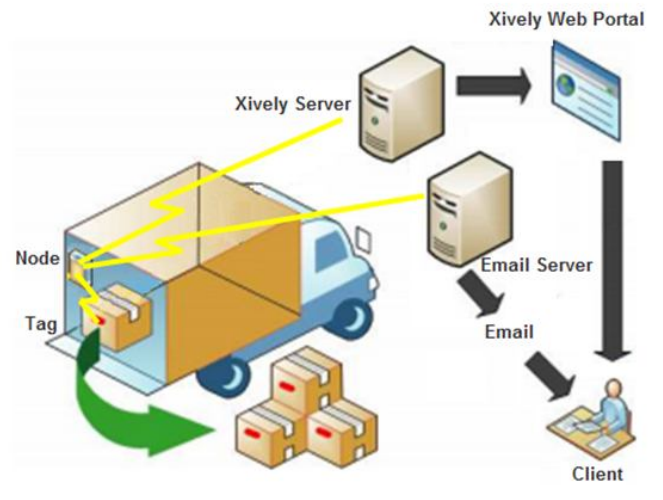

The subsystems are based on Arduino prototyping platform [4]. Each of the system performs these two major tasks: measuring temperature and humidity of the environment and monitoring items moving in and out. On the cloud, the data from facilities follow different paths in reaching the 
monitoring personnel: the temperature and humidity data is uploaded to Xively sensor cloud [5] where it is accessed from the web portal and the inventory information is emailed through the email server.

\subsection{Storage Facility Subsystem}

The storage facilities are warehouses and units at manufacturing facility and distribution sectors that are used for storing the products. The monitoring system for these facilities is composed of two devices: Nodes and Base Station. Node is placed in every storage chamber to be monitored in the facility. The data from the node is transferred to the base station using ZigBee protocol. Base station collects all data and sends it to cloud.

\subsubsection{Node}

The hardware components of the node are Arduino Uno microcontroller, NFC/RFID shield [6], Series2 XBee radio and DHT11 temperature and humidity sensor. External circuit is built-in with the node comprising LCD screen, Piezo Buzzer, LEDs and a Switch. All the hardware components are powered by battery. The LCD screen is used to display measured parameters of the environment on interval basis, Piezo buzzer for local alarm when parameters fall outside the threshold, separate LEDs to indicate successful Tag reads and data transmission to base station and finally the push button switch to read tags. The interval at which the node reads the parameters and transmits it to base station is 5 minutes. Figure 2(a) shows the flowchart of the node algorithm.

Figure 2. (a) Flowchart of Arduino code of Node. (b) Flowchart of Arduino code of Base Station.

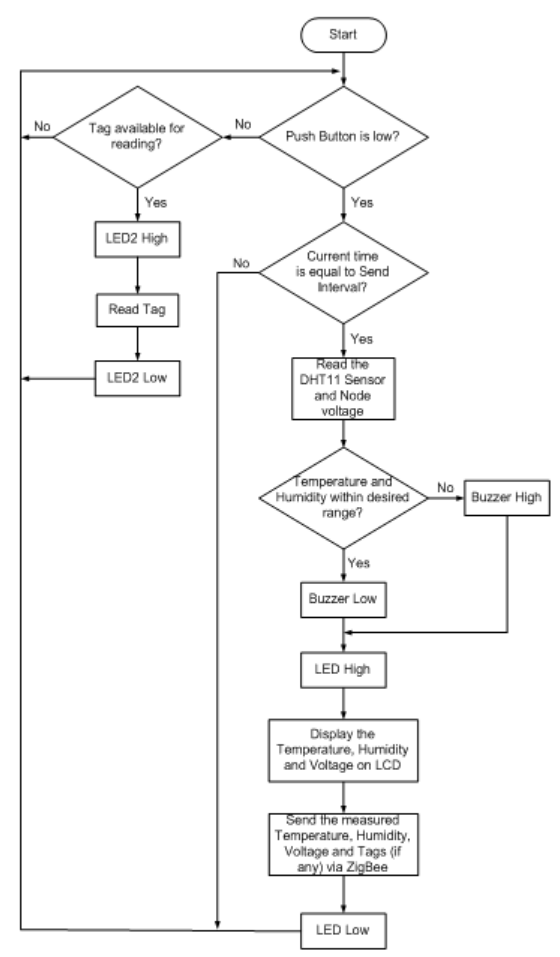

(a)

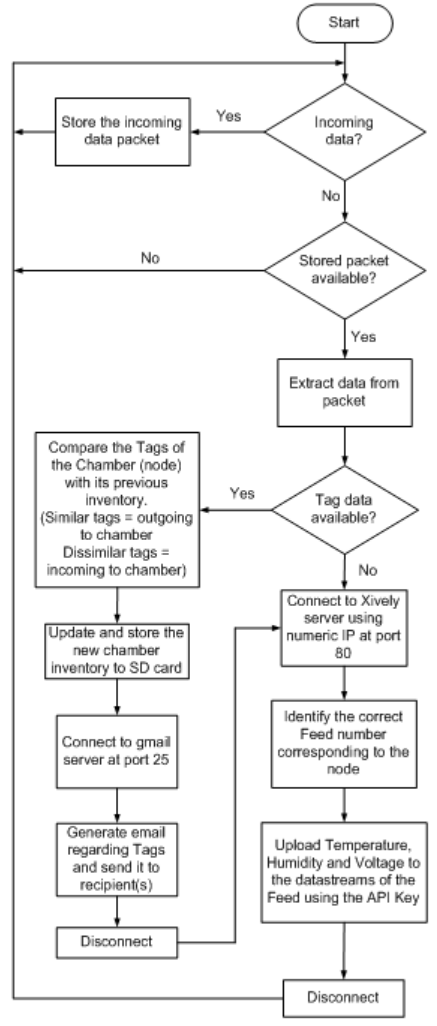

(b) 


\subsubsection{Base Station}

The hardware components of the base station are Arduino Mega microcontroller, Ethernet shield with micro SD card and Series 2 XBee radio. The base station is powered by DC adapter to make sure it is up all the time. Base station processes the received data from nodes and prepares it in order to send it to the cloud. Figure 2(b) shows the flowchart of the algorithm.

\subsection{Transport Facility Subsystem}

Transport facilities play a major role in the cold chain. Monitoring of these logistics is critical since there is a higher risk of product quality compromise due to slow response to an event. The location of carrier from any sort assistance in case of emergency can result in calamity. The monitoring allows notifications to take place or makes aware of any mishap so actions can be taken to prevent its effect on consumers.

The hardware components of the system are Arduino Mega microcontroller, 3G+GPS shield [7], NFC/RFID shield and DHT11 temperature and humidity sensor. Similar to storage monitoring, additional components of this system are LEDs and a push button switch. The push button is used for reading tags which are associated with items. The LEDs indicate processes taking place in the system. The white LED lights up when tag read is successful and blue LED lights up while microcontroller connects and uploads data to cloud. The subsystem is powered via battery. Figure 3 depicts the monitoring process.

Figure 3. Flowchart of the transport facility monitoring system.

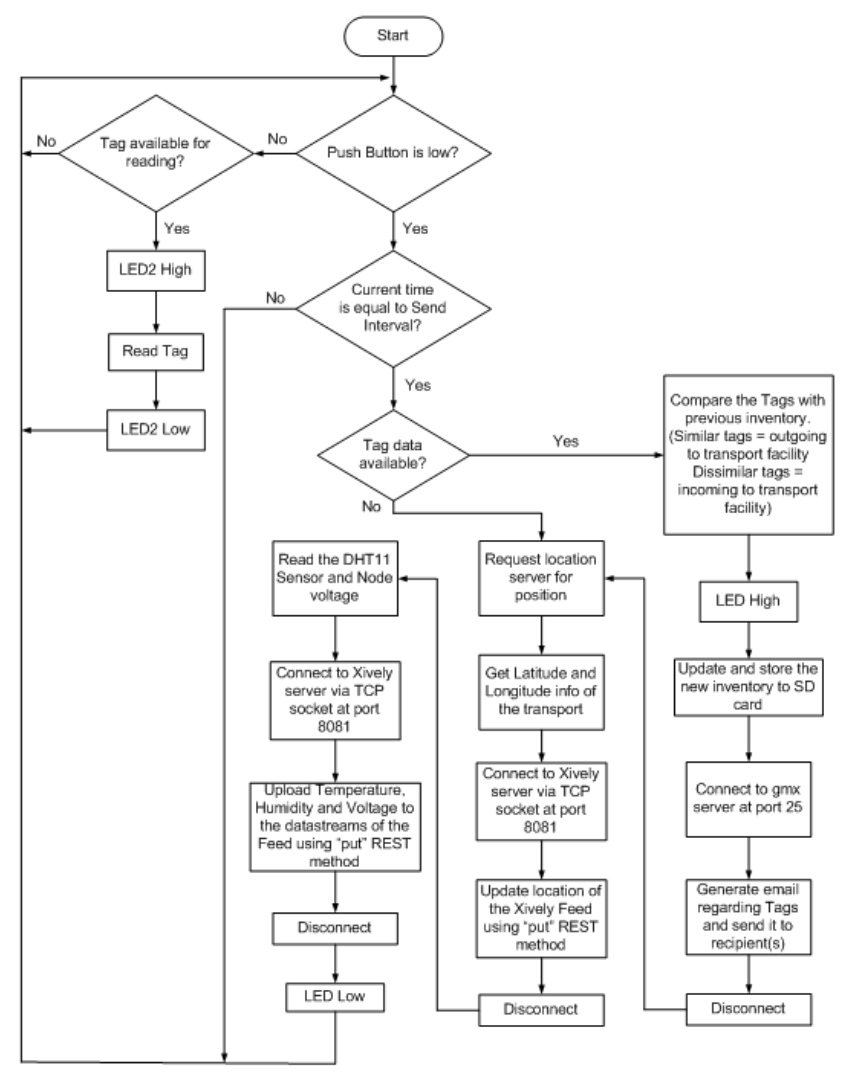




\section{Results and Discussion}

The cold chain monitoring systems measure the temperature and humidity of the environment and upload it to Xively sensor cloud where it is monitored. Besides the environmental parameters, voltage level of the battery is also uploaded to monitor the power level at the nodes and transport facility. On Xively, the data is updated in real-time. Xively also allows setting up of triggers to send notifications about abnormal events. Zapier service [8] was used with Xively to configure applications through which notifications are sent. The application used here was email service.

The system also monitors the inventory of the environment. The tags read through NFC/RFID shield is emailed to the monitoring personnel to provide up-to-date information regarding the items present in the facilities.

Figure 4. Xively web portal.

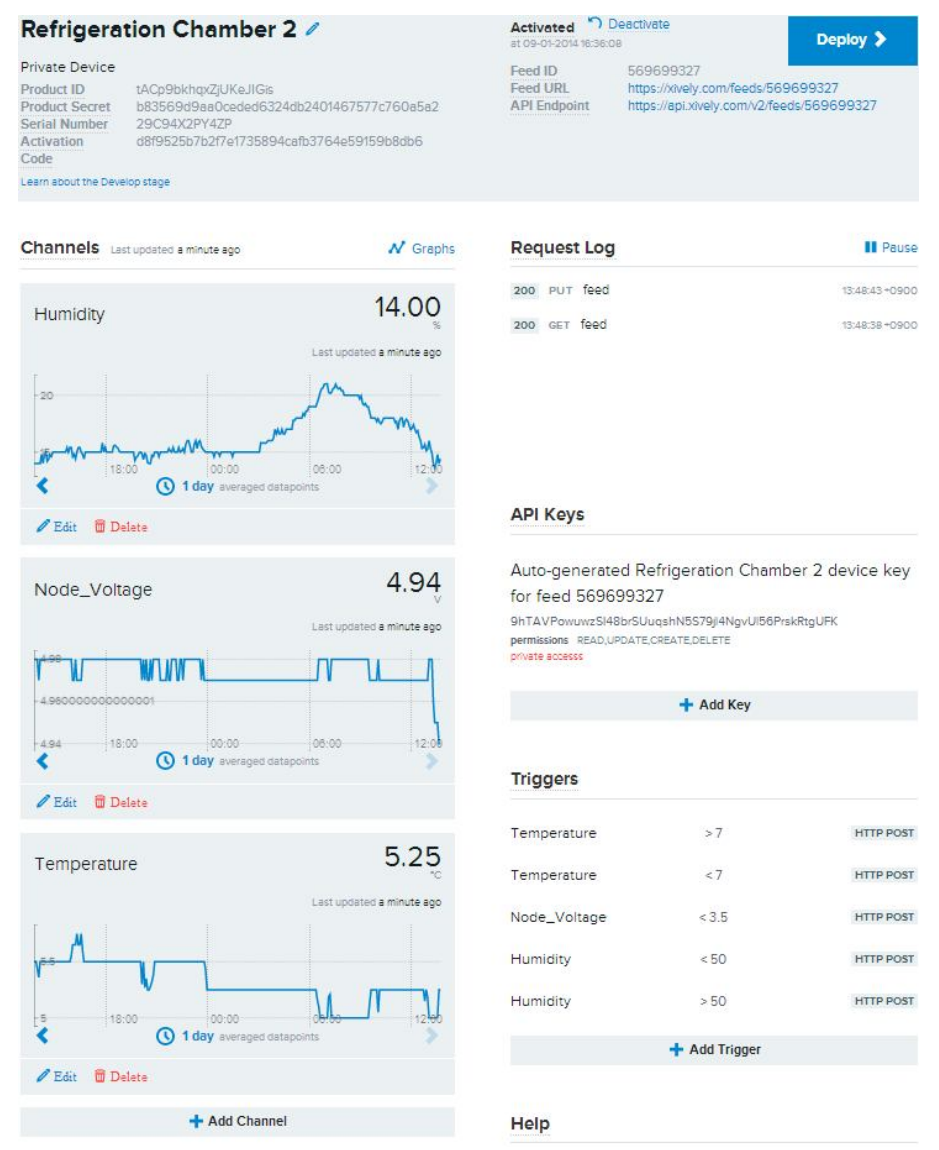

Figure 5(a) shows a sample email trigger for node voltage of refrigeration chamber 1 . The texts in the email are customized using Zapier to develop rich messages. Figure 5(b) illustrates the inventory email of Carrier 1 transport facility. 
Figure 5. (a) Sample email trigger for supply voltage at Chamber 1 in Storage facility. (b)

Carrier 1 inventory email.

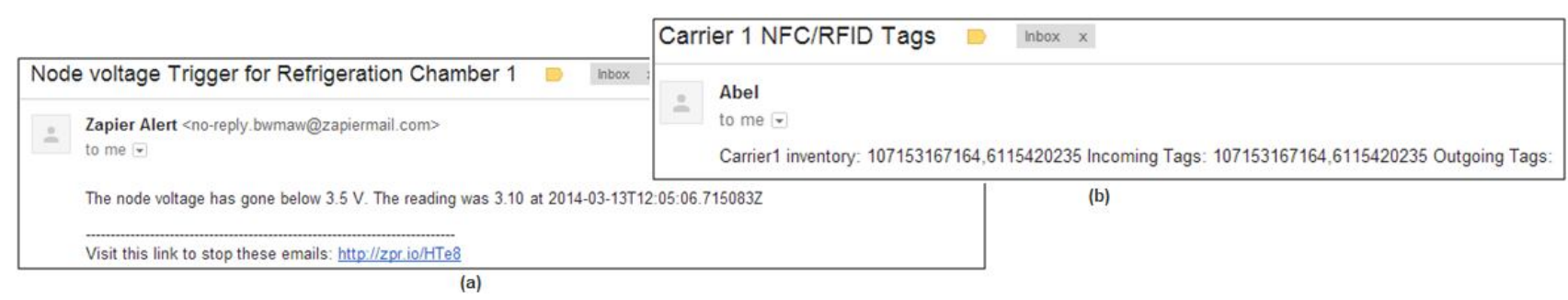

\section{Conclusion}

This work has proposed a cold chain monitoring system that takes advantage of the Internet of Things to provide an improved and enhanced way to monitor the chain. Wireless sensor network with Arduino has been created and interfaced with the Xively sensor cloud where data is uploaded and monitored. The integration has been possible through Arduino libraries and hardware designs for connecting to the internet and web 2.0 technology. The integration brings with it many advantages and benefits which takes the monitoring to a new level. This system not only offers the solution to a problem but lays the foundation for future projects on automating things using IoT.

\section{Conflicts of Interest}

The authors declare no conflict of interest.

\section{References and Notes}

1. Cold Chain Management. cold chain management; Available from: http://ccm.ytally.com/index.php?id=7.

2. The Cold Chain. Public Health Agency of Canada 2012; Available from: http://www.phacaspc.gc.ca/publicat/2007/nvshglp-ldemv/section1-eng.php.

3. Doukas, C., Building Internet of Things with the Arduino2012, USA. 352.

4. Arduino Home Page. Available from: http://www.arduino.cc/.

5. Xively by LogMeln. Available from: https://xively.com/.

6. Adafruit PN532 NFC/RFID controller shield for Arduino + Extras. adafruit; Available from: https://www.adafruit.com/products/789.

7. Libelium. $3 G+G P S$ shield for Arduino. cooking hacks; Available from: http://www.cookinghacks.com/documentation/tutorials/arduino-3g-gprs-gsm-gps\#step14.

8. Zapier. Available from: https://zapier.com/developer/.

(C) 2014 by the authors; licensee MDPI, Basel, Switzerland. This article is an open access article distributed under the terms and conditions of the Creative Commons Attribution license (http://creativecommons.org/licenses/by/3.0/). 